\title{
Dissociating Reading Processes on the Basis of Neuronal Interactions
}

\author{
Andrea Mechelli ${ }^{1}$, Jennifer T. Crinion' ${ }^{1}$, Steven Long ${ }^{1}$, Karl J. Friston ${ }^{1}$, \\ Matthew A. Lambon Ralph ${ }^{2}$, Karalyn Patterson ${ }^{3}$, James L. McClelland ${ }^{4}$, \\ and Cathy J. Price ${ }^{1}$
}

\begin{abstract}
Previous studies of patients with phonological and surface alexia have demonstrated a double dissociation between the reading of pseudo words and words with atypical spelling-tosound relationships. A corresponding double dissociation in the neuronal activation patterns for pseudo words and exception words has not, however, been consistently demonstrated in normal subjects. Motivated by the literature on acquired alexia, the present study contrasted pseudo words to exception words and explored how neuronal interactions within the reading system are influenced by word type. Functional magnetic resonance imaging was used to measure neuronal responses during reading in 22 healthy volunteers. The direct comparison of reading pseudo words and exception words revealed a double dissociation within the left frontal cortex. Pseudo words preferentially increased left
\end{abstract}

\section{INTRODUCTION}

All theories about reading assume that, in translating an alphabetic letter string or word into a pronunciation, readers employ both word-specific knowledge and general, statistical information about the way in which combinations of letters typically correspond to phonological representations. The general information enables pronunciation of words that the reader has never encountered before (called pseudo words in experimental investigations). Word-specific knowledge not only permits comprehension of the words to be read but also contributes to achieving the correct pronunciation of words that violate statistically typical print-sound correspondences: irregular or exception words in English like PINT or STEAK. In the triangle model of reading (Plaut, McClelland, Seidenberg, \& Patterson, 1996), the knowledge base that underlies direct computation from orthography to phonology $(\mathrm{O} \rightarrow \mathrm{P})$ is a combination of

\footnotetext{
${ }^{1}$ Functional Imaging Laboratory, Wellcome Department of Imaging Neuroscience, Institute of Neurology, London, UK, ${ }^{2}$ University of Manchester, UK, ${ }^{3} \mathrm{MRC}$ Cognition and Brain Sciences Unit, Cambridge, UK, ${ }^{4}$ Center for the Neural Basis of Cognition, Pittsburgh, PA, USA
}

dorsal premotor activation, whereas exception words preferentially increased left pars triangularis activation. Critically, these areas correspond to those previously associated with phonological and semantic processing, respectively. Wordtype dependent interactions between brain areas were then investigated using dynamic causal modeling. This revealed that increased activation in the dorsal premotor cortex for pseudo words was associated with a selective increase in effective connectivity from the posterior fusiform gyrus. In contrast, increased activation in the pars triangularis for exception words was associated with a selective increase in effective connectivity from the anterior fusiform gyrus. The present investigation is the first to identify distinct neuronal mechanisms for semantic and phonological contributions to reading. general and word-specific information. Because this knowledge is gradually built up from the reader's experience of printed-word vocabulary, the $\mathrm{O} \rightarrow \mathrm{P}$ computation is most efficient at dealing with regular words (like MINT) and high-frequency exception words (like HAVE); it also supports generalization to pseudo words like RINT or MAVE. It is least efficient at computing the pronunciation of letter strings that benefit neither from high familiarity nor from shared structure with typical words: lower-frequency exception words like PINT. For such words, it is hypothesized that an additional wordspecific contribution from word meaning supports the retrieval of the correct phonological representation, counteracting the influence of domain-general knowledge that would push the reader towards pronouncing PINT as if it rhymed with MINT, PRINT, HINT, LINT, and so forth.

By this account, it is not surprising, and is indeed predicted, that patients with semantic impairments almost invariably have surface alexia, in which reading aloud of regular words, pseudo words, and very common exception words is accurate, but lower-frequency exception words such as PINT are frequently mispronounced as if they were regular. The purest cases of 
surface alexia are patients with semantic dementia, a neurodegenerative syndrome resulting from focal anterior temporal-lobe atrophy and characterized by semantic but not phonological deficits (Hodges, Patterson, Oxbury, \& Funnell, 1992; Patterson \& Hodges, 1992). The contrasting pattern of phonological alexia, typically consequent on infarction of left MCA territory (Lambon Ralph \& Graham, 2000), is seen in patients with prominent phonological, but not semantic, deficits. Their success in reading aloud is predicted not by the spellingsound typicality of the letter string but rather by its lexical/semantic status: Familiar words, especially those with rich imageable meanings, are usually read correctly, but even very simple pseudo words yield very low success rates (Patterson, Suzuki, \& Wydell, 1996; Patterson \& Marcel, 1992). In each of these two contrasting acquired reading disorders, the level of performance on the impaired word type correlates with other nonreading measures of the more general language component assumed to be responsible for the reading deficit. That is, pseudo-word reading difficulties correlate with phonological impairment in phonological alexia (Crisp \& Lambon Ralph, in press) and exception-word reading impairment correlates with degree of semantic degradation in surface alexia (Graham, Patterson, \& Hodges, 2000; Patterson \& Hodges, 1992).

Previous functional neuroimaging studies in normal readers have attempted to identify different neuronal mechanisms for the translation of $\mathrm{O} \rightarrow \mathrm{P}$ by comparing different word types (Mechelli, Gorno-Tempini, \& Price, 2003; Fiebach, Friederici, Müller, \& von Cramon, 2002; Xu et al., 2001; Mechelli, Friston, \& Price, 2000; Paulesu et al., 2000; Tagamets, Novick, Chalmers, \& Friedman, 2000; Fiez, Balota, Raichle, \& Petersen, 1999; Hagoort et al., 1999; Herbster, Mintum, Nebes, \& Becker, 1997; Rumsey et al., 1997; Price, Wise, \& Frackowiak, 1996). To date, however, most of these studies have accrued evidence only for the equivalent of a single, not a double, dissociation. That is, pseudo words relative to real words typically yield increased activation in the left posterior fusiform and left frontal operculum, but no consistent regional activation has been observed for familiar relative to pseudo words (although see Binder et al., 2003). We hypothesize that this null effect might reflect the choice of stimulus materials in these experiments. As noted above, the reading impairment in surface alexia is most striking for lower-frequency exception words, but most functional imaging studies have used high- as well as low-frequency words, and sometimes only those with fairly regular spelling-sound correspondences. Furthermore, functional specialization is not an intrinsic property of a cortical area, but rather depends on both forward and backward connections within a distributed network (Friston \& Price, 2001; McIntosh, 2000; Mesulam, 1990; Damasio, 1989). Thus, differential processing of exception and pseudo words may be reflected not by single regionally selective activations but more by changes in the coupling among different regions within a shared processing system (Price, Gorno-Tempini, et al., 2003; Patterson \& Lambon Ralph, 1999; Plaut et al., 1996). Critically, the subtraction analyses employed in most functional imaging studies have not allowed word-type-selective neuronal interactions to be explored.

The present investigation compared pseudo words with low-frequency words with typical or atypical spelling-tosound correspondences and explored the coupling between different regions of the reading system as a function of word type. Neuronal responses to different word types were measured using functional magnetic resonance imaging (fMRI) in 22 healthy volunteers. A baseline condition (viewing meaningless false fonts) was included to allow assessment of activation that was common to all word types. First, we used statistical parametric mapping to identify the set of regions responsive during reading and to determine how single regional activations were affected by word type. We then explored changes in the coupling between different regions of the reading system as a function of word type, using dynamic causal modeling or DCM (Mechelli, Price, Friston, \& Ishai, 2004; Friston, Harrison, \& Penny, 2003; Mechelli, Price, Noppeney, \& Friston, 2003). DCM treats the brain as a dynamic input-state-output system. The inputs correspond to conventional stimulus functions that encode the experimental factors. The state variables comprise mean synaptic activities and other biophysical variables that determine the outputs. The outputs are the regional hemodynamic responses that are measured using fMRI. In DCM, an experiment is regarded as a designed perturbation of neuronal dynamics that is propagated throughout a network of interconnected anatomical nodes. The coupling between regions is estimated by perturbing the system using a series of inputs and then measuring the changes in regionally specific hemodynamic responses.

In DCM, a reasonably realistic but simple neuronal model of interacting neural regions is constructed, supplemented with a description of how synaptic activity is transformed into a hemodynamic response (see Mechelli, Price, \& Friston, 2001; Friston, Mechelli, Turner, \& Price, 2000). DCM affords inferences about neurodynamics because it employs a forward model, relating neuronal activity to the observed fMRI data. Three sets of parameters are estimated: (i) input parameters which describe how responsive brain regions are to experimental stimuli, (ii) intrinsic parameters that characterize the coupling between regions irrespective of stimulus type, and (iii) modulatory parameters that characterize changes in associated activity caused by experimental manipulation (e.g., regular vs. exception words).

In the present study, we used DCM to test the hypothesis that responses to regular, exception, and pseudo words observed in different regions of the left prefrontal cortex are associated with word-type-sensitive 
connectivity with distinct posterior temporal regions (Price, Gorno-Tempini, et al., 2003). This hypothesis was motivated by the following observations. First, in the left prefrontal cortex, a double dissociation has been observed in ventral and dorsal regions for semantic and phonological tasks, respectively (Devlin, Matthews, \& Rushworth, 2003; McDermott, Petersen, Watson, \& Ojemann, 2003; Roskies, Fiez, Balota, Raichle, \& Petersen, 2001). Specifically, the left pars triangularis is activated by semantic more than phonological tasks and the left premotor cortex is activated by phonological more than semantic tasks. If pseudo words and exception words place differential demands on phonological and semantic processes (Patterson \& Lambon Ralph, 1999), then we should also observe a double dissociation in ventral and dorsal prefrontal activation for different word types. Second, in the left fusiform gyrus, distinct response patterns can be observed in the posterior, middle, and anterior regions. The middle part of the fusiform ( $y=-50$ to $y=-60$ in Talairach space) is consistently activated during lexical retrieval tasks such as reading and picture naming (McCandliss, Cohen, \& Dehaene, 2003; Price \& Devlin, 2003; Price \& Friston, 1999). The most posterior aspect $(y=-60$ to -70$)$ is activated in reading more by pseudo words than by familiar words (see Mechelli, Gorno-Tempini, et al., 2003, for a review). In contrast, the anterior fusiform $(y=-20$ to -50$)$ responds during semantic tasks (Noppeney \& Price, 2002; Mummery, Patterson, Hodges, \& Price, 1998), irrespective of the stimulus modality (visual or auditory). It is this area that we would expect to be more engaged by semantic activation during word reading. Only one study (Herbster et al., 1997) to date has reported greater activation in the anterior fusiform for words relative to pseudo words [Talairach coordinates $(x, y, z):-36,-30$, $-24]$, although we can also observe the same effect in the data reported by Brunswick, McCrory, Price, Frith, and Frith (1999) when the statistical threshold is lowered [Talairach coordinates $(x, y, z)$ : $-30,-26$, $-14 ; Z$ score $=3.4]$. Third, on the basis of functional imaging studies with surface and phonological alexic patients, we have previously proposed that activity in anterior, middle, and posterior fusiform regions may be differentially associated with activation of ventral and dorsal prefrontal regions during reading, and this selective coupling may differ according to word type (Price, Gorno-Tempini, et al., 2003).

A previous fMRI study by Bokde, Tagamets, Friedman, and Horwitz (2001) reported a significant functional correlation between the dorsal inferior frontal gyrus and occipito-temporal regions for orthographic stimuli (i.e., words, pseudo words, and letter strings), but not for false fonts. In contrast, a strong correlation was detected between the ventral inferior frontal gyrus and occipito-temporal regions for meaningful stimuli (i.e., words), but not for pseudo words, letter strings, and false fonts. The authors did not, however, attempt to distinguish between different posterior temporal areas. To test our hypothesis that regular, exception, and pseudo words would yield distinct patterns of effective connectivity between different left posterior temporal regions and left prefrontal regions, we constructed a dynamic causal model of interacting neural regions based on our previously proposed anatomical model of reading (Price, Gorno-Tempini, et al., 2003). Here, we report regional activations as identified by statistical parametric mapping, and then focus on the neuronal interactions among the different brain regions.

\section{RESULTS}

\section{Statistical Parametric Mapping}

Statistical parametric mapping identified the overall effect of reading relative to baseline and the differential effects of word type (Table 1 and Figure 1). Reading all words relative to viewing false fonts increased neuronal activity in a left-lateralized network which included a number of prefrontal and superior temporal regions ( $p<.05$, corrected for multiple comparisons).

Table 1. Regional Effects as Identified with Statistical Parametric Mapping

\begin{tabular}{|c|c|c|c|c|c|c|c|c|c|}
\hline \multirow{2}{*}{$\frac{\text { Region Location }}{\text { Left pars triangularis }}$} & \multicolumn{3}{|c|}{$\begin{array}{l}\text { Reading > } \\
\text { False Fonts }\end{array}$} & $\begin{array}{c}\text { Exception > } \\
\text { Pseudo Words }\end{array}$ & $\begin{array}{c}\text { Pseudo > } \\
\text { Exception Words }\end{array}$ & \multicolumn{2}{|c|}{$\begin{array}{c}\text { Pseudo > } \\
\text { Regular Words }\end{array}$} & \multicolumn{2}{|c|}{$\begin{array}{c}\text { Exception > } \\
\text { Regular Words }\end{array}$} \\
\hline & -52 & 34 & $4(\mathbf{6 . 0})$ & $-52 \quad 34 \quad 4(\mathbf{5 . 4})$ & & & & & \\
\hline Left pars opercularis & -56 & 10 & $4(\mathbf{5 . 6})$ & & & $-54 \quad 8$ & $18(\mathbf{6 . 5})$ & $\begin{array}{ll}-52 & 2\end{array}$ & $18(\mathbf{6 . 0})$ \\
\hline Left dorsal premotor & -52 & -8 & $44(5.6)$ & & $40(5.6)$ & & & & \\
\hline Left superior frontal & -2 & 2 & $64(5.0)$ & & & & & & \\
\hline Left superior temporal & -56 & -44 & $4(\mathbf{5 . 2})$ & & & & & & \\
\hline Left occipito-temporal & -42 & -46 & $-28(3.9)$ & & & & & & \\
\hline
\end{tabular}

We report region location, coordinates, and $Z$ scores (in parentheses) for the overall effect of reading relative to viewing false fonts and for the differential effects of word type. $Z$ scores in bold are significant at $p<.05$ (corrected for multiple comparisons). $Z$ scores not in bold are significant at $p<.05$ (uncorrected). ns $=$ not significant when lowering the statistical threshold to $p<.05$ (uncorrected). 


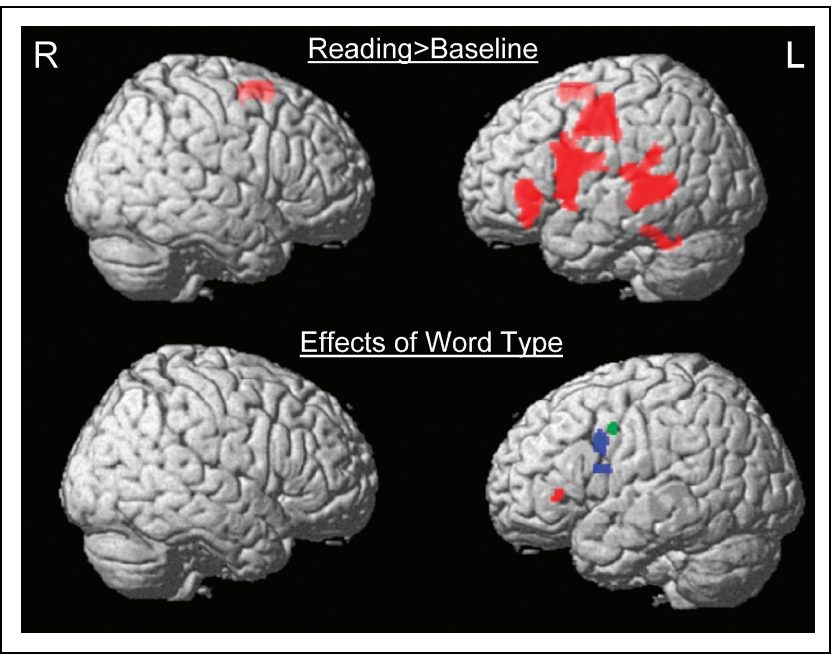

Figure 1. The regional effects detected during reading regular, exception, and pseudo words. Upper row: Brain areas that expressed an overall effect of reading relative to viewing strings of false fonts $(p<.001$, uncorrected). Lower row: Brain areas that responded to regular, exception, and pseudo words differentially $(p<.05$, corrected for multiple comparisons). Red indicates the region in the pars triangularis which responded to exception words more than pseudo words; blue indicates the region in the pars opercularis that responded to both pseudo words and exception words more than regular words; green indicates the region in the dorsal premotor cortex which responded to pseudo words more than exception words.

Three distinct regions within the left prefrontal cortex expressed differential patterns of activation for regular, exception, and pseudo words. The pars triangularis showed increased activation for exception relative to pseudo words $(x=-52, y=32, z=4 ; Z$ score $=5.4)$. The reverse effect was found in the dorsal premotor cortex, which expressed increased activation for pseudo relative to exception words $(x=-56, y=0, z=40 ; Z$ score $=5.6)$. Finally, the pars opercularis showed increased activation for exception relative to regular words $(x=-52, y=2, z=18 ; Z$ score $=6.0)$ and for pseudo relative to regular words $(x=-54, y=8$, $z=18 ; Z$ score $=6.5$ ).

Although differential effects of word type were not found in distinct fusiform regions at the conservative criterion of $p<.05$ (corrected for multiple comparisons), lowering the statistical threshold to $p<.05$ (uncorrected) yielded a number of trends in these regions that were consistent with previous studies. In particular, the left anterior fusiform was more active for exception words than for pseudo words $(x=-42, y=-42, z=$ $-18 ; Z$ score $=3.8$ ), as previously reported by Herbster et al. (1997). In contrast, increased activation in the left posterior fusiform was detected for pseudo words relative to regular words $(x=-46, y=-60, z=-18$; $Z$ score $=2.2$ ) as previously reported by Mechelli, Gorno-Tempini, et al. (2003), Paulesu et al. (2000), and Brunswick et al. (1999) (see Table 1 for details).

\section{Dynamic Causal Modeling}

A dynamic causal model was estimated that included seven left-lateralized regions of interest: pars triangularis, pars opercularis, dorsal premotor cortex, posterior fusiform, middle fusiform, anterior fusiform, and superior temporal cortex. These regions were defined as 6-mm-radius spheres centered on the maxima of subject-specific statistical parametric maps, testing for the overall effect of reading relative to false fonts (i.e., not on the basis of word-type effects). Regional activations were extracted in terms of the principal eigenvariate from each region, in a subject-specific fashion. Note that DCM does not estimate the correlation or covariance between regions but, rather, the strength of the connections in the user-specified model and how this may change from one experimental condition to another. We refer to these values as forward and backward connections. The DCM included forward and backward "intrinsic connections" between the three fusiform areas and the superior temporal area; between the superior temporal area and the three prefrontal areas; and between the three fusiform areas and the three prefrontal areas as represented graphically in Figure 2 .

\section{Intrinsic Connections}

Intrinsic connections refer to the coupling between regions irrespective of stimulus type and can be regarded as a kind of "baseline" network established by the experimental context (i.e., task set). We found a number of forward and backward connections that were

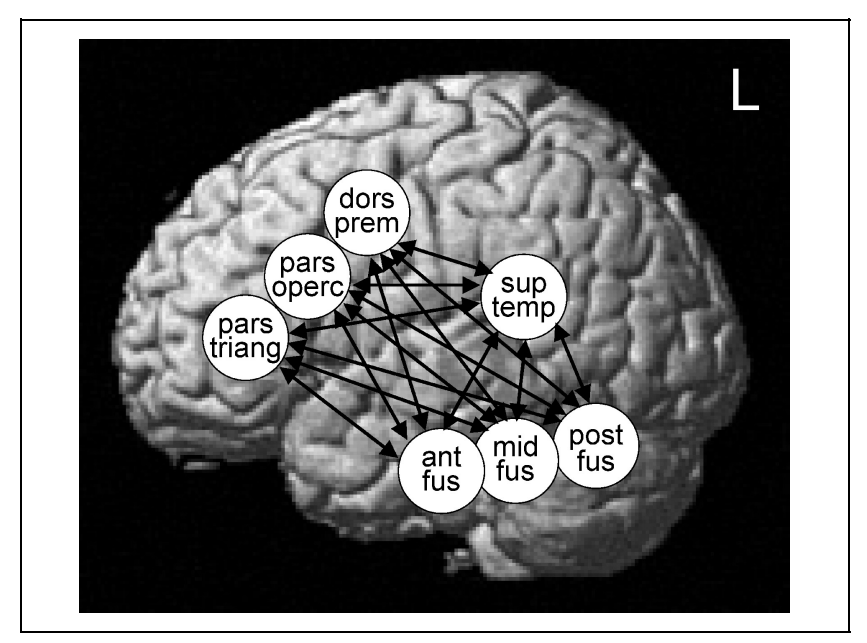

Figure 2. The dynamic causal model that was used to investigate the interregional coupling within the reading system, and how this was influenced by word type. The model included seven left-lateralized regions: pars triangularis (pars triang), pars opercularis (pars operc), dorsal premotor cortex (dors prem), posterior fusiform (post fus), middle fusiform (mid fus), anterior fusiform (ant fus), and superior temporal cortex (sup temp). The bidirectional arrows indicate forward and backward connections between two anatomical nodes. 
significantly different from 0 (see Table 2 and Figure 3A). Specifically, the forward connection between the posterior fusiform and the pars triangularis was significantly lower than $0(t=-4.1, d f=21 ; p<.001)$. In con- trast, the forward connection between activity in the posterior fusiform and dorsal premotor cortex was significantly greater than $0(t=4.8, d f=21 ; p<.001)$. Thus, the posterior fusiform was negatively coupled

Table 2. Estimated Parameters for the Intrinsic Connections, which Refer to the Coupling between Two Regions (X and Y) Irrespective of Word Type

\begin{tabular}{|c|c|c|c|}
\hline Intrinsic Connections & Mean & $S D$ & $p$ \\
\hline \multicolumn{4}{|l|}{ Forward } \\
\hline Posterior fusiform $\rightarrow$ Pars triangularis & -0.089 & 0.098 & $<.001$ \\
\hline Posterior fusiform $\rightarrow$ Pars opercularis & 0.071 & 0.155 & .043 \\
\hline Posterior fusiform $\rightarrow$ Dorsal premotor & 0.156 & 0.151 & $<.001$ \\
\hline Middle fusiform $\rightarrow$ Pars triangularis & 0.060 & 0.091 & .005 \\
\hline Middle fusiform $\rightarrow$ Pars opercularis & 0.048 & 0.100 & .035 \\
\hline Middle fusiform $\rightarrow$ Dorsal premotor & 0.043 & 0.077 & .016 \\
\hline Anterior fusiform $\rightarrow$ Pars triangularis & 0.092 & 0.089 & $<.001$ \\
\hline Anterior fusiform $\rightarrow$ Pars opercularis & -0.011 & 0.111 & .64 \\
\hline Anterior fusiform $\rightarrow$ Dorsal premotor & -0.093 & 0.080 & $<.001$ \\
\hline Posterior fusiform $\rightarrow$ Superior temporal & 0.023 & 0.134 & .423 \\
\hline Middle fusiform $\rightarrow$ Superior temporal & 0.044 & 0.140 & .156 \\
\hline Anterior fusiform $\rightarrow$ Superior temporal & -0.019 & 0.122 & .459 \\
\hline Superior temporal $\rightarrow$ Pars triangularis & 0.014 & 0.053 & .232 \\
\hline Superior temporal $\rightarrow$ Pars opercularis & 0.035 & 0.039 & $<.001$ \\
\hline Superior temporal $\rightarrow$ Dorsal premotor & 0.021 & 0.048 & .047 \\
\hline \multicolumn{4}{|l|}{ Backward } \\
\hline Pars triangularis $\rightarrow$ Posterior fusiform & -0.009 & 0.024 & .103 \\
\hline Pars opercularis $\rightarrow$ Posterior fusiform & 0.006 & 0.022 & .236 \\
\hline Dorsal premotor $\rightarrow$ Posterior fusiform & 0.012 & 0.023 & .024 \\
\hline Pars triangularis $\rightarrow$ Middle fusiform & 0.001 & 0.019 & .849 \\
\hline Pars opercularis $\rightarrow$ Middle fusiform & 0.009 & 0.022 & .048 \\
\hline Dorsal premotor $\rightarrow$ Middle fusiform & 0.011 & 0.031 & .105 \\
\hline Pars triangularis $\rightarrow$ Anterior fusiform & 0.002 & 0.010 & .369 \\
\hline Pars opercularis $\rightarrow$ Anterior fusiform & 0.000 & 0.019 & .874 \\
\hline Dorsal premotor $\rightarrow$ Anterior fusiform & -0.002 & 0.020 & .528 \\
\hline Superior temporal $\rightarrow$ Posterior fusiform & -0.001 & 0.025 & .791 \\
\hline Superior temporal $\rightarrow$ Middle fusiform & 0.004 & 0.033 & .549 \\
\hline Superior temporal $\rightarrow$ Anterior fusiform & -0.001 & 0.012 & .777 \\
\hline Pars triangularis $\rightarrow$ Superior temporal & 0.011 & 0.048 & 0.309 \\
\hline Pars opercularis $\rightarrow$ Superior temporal & 0.036 & 0.038 & $<.001$ \\
\hline Dorsal premotor $\rightarrow$ Superior temporal & 0.024 & 0.045 & .025 \\
\hline
\end{tabular}

Negative mean values indicate that an increase of activity in $\mathrm{X}$ is associated with a decrease in $\mathrm{Y}$; positive mean values indicate that an increase of activity in $\mathrm{X}$ is associated with an increase in Y. Mean coupling parameters over subjects (mean), standard deviation (SD), and two-tailed statistical significance $(p)$ are reported in bold when significant at $p<.001$. 
Figure 3. Neuronal interactions during reading. (Left) Coupling of activation in two distinct regions ( $\mathrm{X}$ and $\mathrm{Y}$ ) irrespective of word type (i.e., "intrinsic connectivity"): negative connection strengths (in blue) indicate that an increase of activity in $\mathrm{X}$ is associated with a decrease in $Y$; positive connection strengths (in red) indicate that an increase of activity in $\mathrm{X}$ is associated with an increase in Y. (Right) Differential effect of word type on the intrinsic connectivity: effects strongest for pseudo words and for exception words are indicated in green and yellow, respectively; no differential effects were detected for regular words.
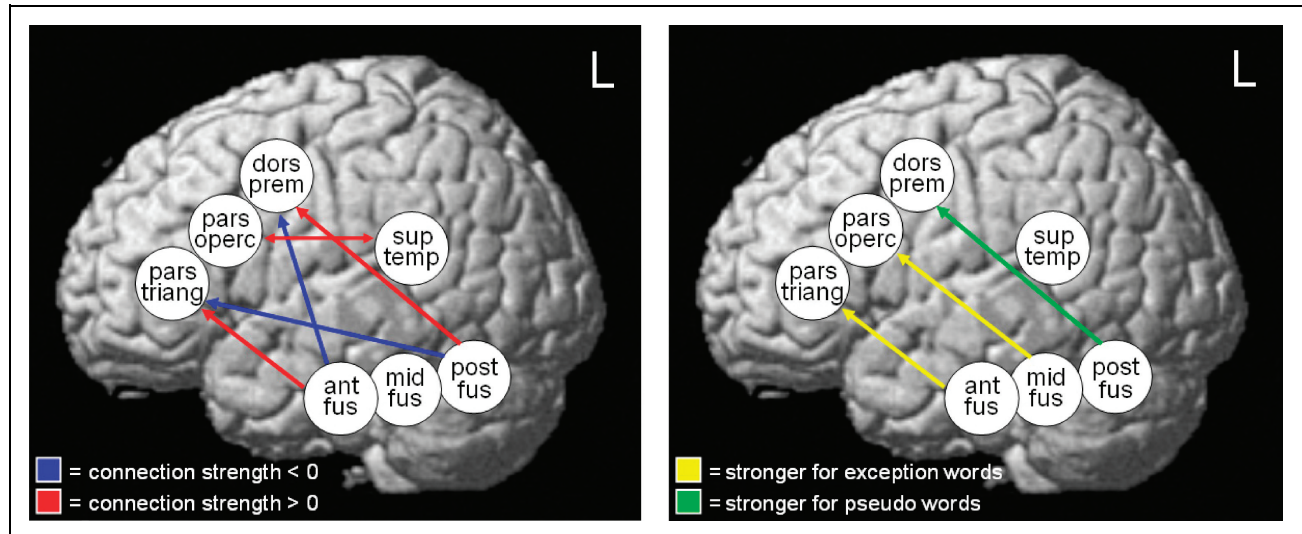

with the pars triangularis but positively coupled with the dorsal premotor cortex. Strikingly, the reverse pattern of intrinsic connectivity applied to the anterior fusiform: The strength of the forward connection from the anterior fusiform to the pars triangularis was significantly greater than $0(t=4.9, d f=21 ; p<.001)$, but the strength of the forward connection from the anterior fusiform to the dorsal premotor cortex was significantly lower than $0(t=-5.4, d f=21 ; p<.001)$. Finally, DCM detected significant forward and backward positive connections $(t=4.2, d f=21 ; p<.001$ and $t=4.4, d f=$ $21 ; p<.001$, respectively) between the superior temporal cortex and the pars opercularis.

\section{Influence of Word Type on the Intrinsic Connections}

As expected, we found a number of intrinsic connections which were significantly modulated by word type (see Table 3 and Figure 3B). Specifically, (i) the forward connection between the posterior fusiform and dorsal premotor cortex was significantly stronger for pseudo words relative to exception and regular words $(t=4.8, d f=21 ; p<.001)$; (ii) the forward connection between the anterior fusiform and pars triangularis was significantly stronger for exception words relative to pseudo and regular words $(t=5.7, d f=$ $21 ; p<.001)$; and (iii) the forward connection between activity in the middle fusiform and pars opercularis was stronger for exception words relative to regular and pseudo words $(t=4.1, d f=21$; $p<.001)$. There were no connections that were significantly stronger for regular than exception and pseudo words. These results are consistent with our prediction that different responses to regular, exception, and pseudo words in the left prefrontal cortex are associated with word-type-sensitive connectivity with occipito-temporal areas.

\section{DISCUSSION}

The aim of the present investigation was to draw inferences about the neuronal network that underlies the complex process of translating orthography to phonology. Specifically, we tested the hypothesis that responses to regular, exception, and pseudo words observed in different regions of the left prefrontal cortex are associated with word-type-sensitive connectivity with distinct posterior temporal regions (Price, GornoTempini, et al., 2003). To address this hypothesis, we examined not only region-specific responses to regular, exception, or pseudo words, but also the impact of word type on the coupling among different components of the reading system.

\section{Regional Effects}

Statistical parametric mapping was used to identify the main effect of reading relative to viewing false fonts and the differential effects of regular, exception, and pseudo words. Strings of real letters relative to false fonts increased activity in a left-lateralized network including prefrontal, superior temporal, and posterior temporal areas. This distributed activation is consistent with previous studies of word and pseudo-word reading (Mechelli, Gorno-Tempini, et al., 2003; Price, GornoTempini, et al., 2003; Turkeltaub, Eden, Jones, \& Zeffiro, 2002; Fiez, Balota, et al., 1999). Within this distributed network, three distinct regions expressed differential sensitivity to regular, exception, and pseudo words. The pars triangularis showed increased activation for 
Table 3. Estimated Parameters for the Bilinear Terms, which Refer to the Influence of Word Type on the Intrinsic Connections

\begin{tabular}{|c|c|c|c|c|c|c|c|c|c|}
\hline \multirow[b]{2}{*}{ Word Type Effects } & \multicolumn{3}{|c|}{ Regular Words } & \multicolumn{3}{|c|}{ Exception Words } & \multicolumn{3}{|c|}{ Pseudo Words } \\
\hline & Mean & $S D$ & $p$ & Mean & $S D$ & $p$ & Mean & $S D$ & $p$ \\
\hline \multicolumn{10}{|l|}{ Forward } \\
\hline Posterior fusiform $\rightarrow$ Pars triangularis & -0.003 & 0.012 & .317 & 0.001 & 0.021 & .814 & -0.009 & 0.017 & .021 \\
\hline Posterior fusiform $\rightarrow$ Pars opercularis & 0.002 & 0.010 & .419 & 0.002 & 0.011 & .404 & 0.008 & 0.021 & .064 \\
\hline Posterior fusiform $\rightarrow$ Dorsal premotor & 0.000 & 0.012 & .930 & 0.003 & 0.009 & .067 & 0.023 & 0.022 & $<.001$ \\
\hline Middle fusiform $\rightarrow$ Pars triangularis & 0.003 & 0.010 & .163 & 0.000 & 0.015 & .932 & -0.002 & 0.008 & .249 \\
\hline Middle fusiform $\rightarrow$ Pars opercularis & 0.002 & 0.008 & .307 & 0.005 & 0.005 & $<.001$ & 0.002 & 0.015 & .387 \\
\hline Middle fusiform $\rightarrow$ Dorsal premotor & 0.002 & 0.004 & .025 & -0.001 & 0.008 & .460 & 0.008 & 0.019 & .058 \\
\hline Anterior fusiform $\rightarrow$ Pars triangularis & 0.003 & 0.010 & .213 & 0.020 & 0.016 & $<.001$ & -0.001 & 0.011 & .809 \\
\hline Anterior fusiform $\rightarrow$ Pars opercularis & 0.003 & 0.008 & .171 & 0.003 & 0.004 & .021 & -0.006 & 0.019 & .150 \\
\hline Anterior fusiform $\rightarrow$ Dorsal premotor & 0.002 & 0.007 & .176 & -0.004 & 0.012 & .082 & -0.011 & 0.019 & .015 \\
\hline Posterior fusiform $\rightarrow$ Superior temporal & 0.000 & 0.011 & .848 & 0.001 & 0.012 & .789 & 0.002 & 0.011 & .260 \\
\hline Middle fusiform $\rightarrow$ Superior temporal & 0.003 & 0.007 & .079 & 0.004 & 0.008 & .027 & 0.000 & 0.013 & .897 \\
\hline Anterior fusiform $\rightarrow$ Superior temporal & 0.002 & 0.013 & .482 & 0.003 & 0.004 & .004 & -0.006 & 0.011 & .023 \\
\hline Superior temporal $\rightarrow$ Pars triangularis & 0.001 & 0.003 & .109 & 0.003 & 0.004 & .002 & -0.003 & 0.003 & .001 \\
\hline Superior temporal $\rightarrow$ Pars opercularis & 0.000 & 0.003 & .803 & 0.001 & 0.002 & .190 & 0.003 & 0.004 & .002 \\
\hline Superior temporal $\rightarrow$ Dorsal premotor & 0.000 & 0.003 & .459 & -0.002 & 0.003 & .016 & 0.004 & 0.005 & .002 \\
\hline \multicolumn{10}{|l|}{ Backward } \\
\hline Pars triangularis $\rightarrow$ Posterior fusiform & 0.000 & 0.001 & .075 & -0.001 & 0.001 & .106 & 0.000 & 0.001 & .945 \\
\hline Pars opercularis $\rightarrow$ Posterior fusiform & 0.000 & 0.001 & .162 & -0.001 & 0.002 & .060 & 0.000 & 0.001 & .207 \\
\hline Dorsal premotor $\rightarrow$ Posterior fusiform & 0.000 & 0.001 & .211 & -0.001 & 0.002 & .257 & 0.000 & 0.001 & .239 \\
\hline Pars triangularis $\rightarrow$ Middle fusiform & 0.000 & 0.002 & .189 & 0.000 & 0.001 & .801 & 0.000 & 0.001 & .685 \\
\hline Pars opercularis $\rightarrow$ Middle fusiform & 0.000 & 0.001 & .294 & -0.001 & 0.002 & .858 & 0.000 & 0.001 & .925 \\
\hline Dorsal premotor $\rightarrow$ Middle fusiform & 0.000 & 0.001 & .385 & 0.000 & 0.002 & 612 & 0.000 & 0.002 & .599 \\
\hline Pars triangularis $\rightarrow$ Anterior fusiform & 0.000 & 0.001 & .195 & 0.000 & 0.001 & .480 & 0.000 & 0.001 & 621 \\
\hline Pars opercularis $\rightarrow$ Anterior fusiform & 0.000 & 0.001 & .758 & 0.000 & 0.001 & .719 & 0.000 & 0.001 & .152 \\
\hline Dorsal premotor $\rightarrow$ Anterior fusiform & 0.000 & 0.001 & .389 & 0.001 & 0.001 & .281 & -0.001 & 0.001 & .094 \\
\hline Superior temporal $\rightarrow$ Posterior fusiform & 0.000 & 0.001 & .107 & -0.001 & 0.001 & .013 & 0.001 & 0.002 & .169 \\
\hline Superior temporal $\rightarrow$ Middle fusiform & 0.000 & 0.002 & .077 & 0.000 & 0.002 & .792 & 0.000 & 0.002 & .888 \\
\hline Superior temporal $\rightarrow$ Anterior fusiform & 0.000 & 0.001 & .532 & 0.000 & 0.001 & .815 & 0.000 & 0.001 & .341 \\
\hline Pars triangularis $\rightarrow$ Superior temporal & 0.001 & 0.003 & .277 & 0.001 & 0.002 & .248 & -0.001 & 0.002 & .174 \\
\hline Pars opercularis $\rightarrow$ Superior temporal & 0.001 & 0.002 & .045 & 0.001 & 0.003 & .054 & 0.001 & 0.003 & .116 \\
\hline Dorsal premotor $\rightarrow$ Superior temporal & 0.000 & 0.002 & .538 & 0.001 & 0.003 & .185 & 0.001 & 0.004 & .234 \\
\hline
\end{tabular}

Negative mean values indicate word-type-related decreases in the strength of the coupling; positive mean values indicate word-type-related increases in the strength of coupling. Mean parameters over subjects (mean), standard deviation $(S D)$, and two-tailed statistical significance $(p)$ are reported in bold when significant at $p<.001$. 
exception relative to pseudo words, whereas the dorsal premotor cortex revealed increased activation for pseudo relative to exception words. Critically, this contrast parallels the double dissociation between semantic and phonological processing previously reported in the very same regions (Devlin et al., 2003; McDermott et al., 2003; Roskies et al., 2001). In addition, the pars opercularis expressed increased activation for both exception and pseudo words relative to regular words. The pattern of response in the pars opercularis replicates previous studies that compared regular, exception, and pseudo words (Fiez, Balota, et al., 1999; Hagoort et al., 1999; Herbster et al., 1997; Rumsey et al., 1997). Other functional neuroimaging studies have suggested the involvement of the pars opercularis in a range of phonological tasks, some of which do not require orthographic to phonological $(\mathrm{O} \rightarrow \mathrm{P})$ translation (Fiez, Katz, \& Tallal, 1995; Zatorre, Evans, Meyer, \& Gjedde, 1992). Furthermore, frontal lesions are typically observed in patients with acquired phonological dyslexia whose poor pseudo word reading is hypothesized to result from their more general phonological deficits (Crisp \& Lambon Ralph, in press; Patterson et al., 1996; Patterson \& Marcel, 1992). Thus, this region may contribute to the effortful selection, retrieval, and/or manipulation of phonological representations critical for reading and other phonological tasks as proposed by Fiez, Balota, et al. (1999).

The findings regarding the pars triangularis and the dorsal premotor cortex extend to those reported in previous functional neuroimaging studies by demonstrating a double dissociation in activation for exception and pseudo words. This double dissociation suggests that our results cannot simply be attributed to increased difficulty or intersubject variability for one word type relative to another. These differences in the amplitude of single regional responses, however, provide only limited information about the neuronal network responsible for reading or any other complex cognitive function. In this instance, the varying patterns of left prefrontal activation are probably associated particularly with the phonological "end" of the $\mathrm{O} \rightarrow \mathrm{P}$ computation, whereas our goal was to illuminate this whole computation. We therefore used DCM to determine how various prefrontal regions were coupled with areas more likely to be associated with the orthographic stage of this computation, namely, the posterior, middle, and anterior fusiform regions.

\section{Regional Coupling}

The DCM analysis of interregional coupling revealed a double dissociation between the posterior and anterior fusiform: The former was negatively coupled to the pars triangularis but positively coupled with the dorsal premotor cortex; the latter was positively coupled to the pars triangularis but negatively coupled with the dorsal premotor cortex. However, the primary focus of our DCM was the influence of word type on the coupling among regions. For pseudo words, the estimate of connectivity in our DCM model revealed an increase in coupling between the dorsal premotor cortex and the posterior fusiform. For exception words, increased activation in the pars triangularis was associated with a selective increase in connectivity from the anterior fusiform, whereas increased activation in the pars opercularis was associated with a selective enabling of connections from the middle fusiform. Thus, differential responses to regular, exception, and pseudo words in the prefrontal cortex were associated with word-typesensitive coupling with distinct left occipito-temporal areas.

It should be emphasized that DCM does not assume direct anatomical connections between regions of interest: Regional couplings can be investigated with DCM, even when the underlying anatomical connections are indirect and polysynaptic. For instance, the significant association between responses in the posterior fusiform and the dorsal premotor cortex during pseudo word reading, or between the anterior fusiform and the pars triangularis during exception word reading, may be mediated via regions not included in the model. In fact, this seems rather likely to be the case when the coupled regions are as far apart as these particular pairs of brain areas. If this is true, then it might be of interest to know about associated activity in the other intervening area(s); but such additional information does not reduce the theoretical interest afforded by the functional coupling observed here. The combination of a priori bases for our predictions plus stringent statistical tests on our data makes it highly unlikely that the observed associations are accidental. Even if other brain regions are involved, therefore, it seems a reasonable interpretation that the coupled areas are functionally, meaningfully associated.

Our findings are consistent with the results of a previous functional neuroimaging study that investigated interregional coupling within the reading system: Bokde et al. (2001) reported that dorsal and ventral inferior frontal cortices expressed differential connectivity with posterior brain regions, but these authors did not attempt to distinguish between different occipitotemporal areas or to dissociate pathways for translating orthography to phonology at the neuronal level. Our study significantly extends their findings by showing that differential neuronal responses in contiguous prefrontal regions covary with activity in distinct fusiform areas. Indeed, close inspection of the coordinates reported by Bokde et al. reveals that activity in the ventral inferior frontal gyrus correlated with that in an anterior fusiform area $(x=-48, y=-44, z=-20)$, whereas the dorsal inferior frontal gyrus correlated with a middle fusiform area $(x=-42, y=-54, z=-24)$. 
Our results are also fully consistent with the contemporary theoretical approach to reading and acquired alexia that partly inspired this imaging investigation (Patterson \& Lambon Ralph, 1999; Plaut et al., 1996), although it should be emphasized that nothing in the imaging study was actually designed to discriminate between the predictions of this particular model of reading and other more strictly dual-route models such as Coltheart et al. (2001). According to the approach favored here, the double dissociation of acquired phonological and surface dyslexia reflects selective reductions in the contribution of underlying phonological and semantic representations, respectively. Our imaging data suggest a parallel distinction between the contributions from these basic phonological and semantic components of the language system to pseudo-word and exception-word reading in normal readers. It is important to note the following point about the patients with semantic dementia who demonstrate such a striking pattern of surface alexia: The atrophy causing their profound semantic degradation is most prominent in the temporal polar cortex (i.e., even more anterior than the anterior fusiform whose coupling with the pars triangularis in this study was notable specifically for exception words). This "shift" from the anterior fusiform to the temporal pole may not be so critical, however, in light of a PET study of patients with semantic dementia performing a relatively simple semantic task (Mummery et al., 1998). This study revealed decreased activation for the patients relative to controls in the posterior inferior temporal cortex, where there is no apparent structural abnormality for the patients (Mummery et al., 1998). That observation suggests that this more posterior area normally receives feedback from the temporal polar region that is so abnormal in semantic dementia; in other words, these areas too might normally be subject to coupled activation.

In summary, our investigation was motivated by the idea that word-type sensitivity is not an intrinsic property of a cortical area but, rather, depends on differential forward and backward connections within the reading system. Our findings illustrate that different patterns of activations for regular, exception, and pseudo words in the left prefrontal cortex are associated with activity in distinct left posterior temporal regions. Our working hypothesis regarding the implications of the results for an understanding of the translation of orthography to phonology at both the cognitive and the neural level is as follows. First, although access to at least some aspects of word meaning during this translation process probably occurs for all real words, the semantic contribution is more important for pronunciation of exception than regular words, and is reflected in augmented coupling between the anterior fusiform and the pars triangularis. Second, although phonological processing is obviously required for any type of letter string, this phonological contribution serves a more demanding role in pronouncing pseudo words than real words, and is reflected in greater coupling between the posterior fusiform and the dorsal premotor cortex. Although aspects of these working hypotheses have been proposed in previous publications (Price, Gorno-Tempini, et al., 2003; Patterson \& Lambon Ralph, 1999; Plaut et al., 1996), they receive direct support from the DCM techniques employed here. This support would not be achievable with any other experimental approach, including conventional analyses of fMRI activations.

\section{METHODS}

\section{Subjects}

Informed consent was obtained from 22 right-handed volunteers (12 men and 10 women), aged between 21 and 54 years (with a mean age of 36), with English as their first language. None reported a history of neurological or psychiatric illness, or disturbances in speech comprehension, speech production, reading, or writing.

\section{Experimental Paradigm}

The experiment used four types of letter or letter-like strings, varying in length from 4 to 10 elements: real English words with regular/typical spelling-sound correspondences (e.g., cult, shock, fact, chill); real English words with exceptional/atypical spelling-sound correspondences (e.g., folk, debt, doubt, cough); English pseudo words (e.g., welck, tholt, hoosh, genck); and strings of false fonts, composed of nonorthographic symbols that matched real letters in terms of visual complexity. Regular words, exception words, and pseudo words were matched for number of letters, number of syllables, and bigram frequency. In addition, regular words and exception words were matched for familiarity (Coltheart, 1981), imageability (Coltheart, 1981), and written frequency (Kucera \& Francis, 1967). Written frequency (per million) ranged from 1 to 447 with a mean of 40.8 and a standard deviation of 66.48 .

Two similar experimental parameters were used for stimulus presentation. Eleven subjects (aged between 21 and 39 , mean age 28 years) were presented with stimuli of the same type in blocks of $21 \mathrm{sec}$, with a stimulus onset asynchrony (SOA) of $3 \mathrm{sec}$ and an exposure duration of $750 \mathrm{msec}$. The remaining 11 subjects (aged between 36 and 54, mean age 46 years) were presented with stimuli of the same type in blocks of $20 \mathrm{sec}$, with an SOA of $4 \mathrm{sec}$ and an exposure duration of $3 \mathrm{sec}$. Because no between-group differences were observed, data from all 22 subjects were pooled together using a randomeffects statistical model. This means that any significant regional effects and neuronal interactions occurred irrespective of stimulus presentation and age (Holmes \& 
Friston, 1998). Subjects were instructed to read each alphabetical letter string silently as soon as it appeared on the screen and to look at the false font strings. To ensure that the subjects had attended to the stimuli, an eye-tracker was used to monitor eye movements.

\section{Scanning Technique}

A Siemens $1.5-\mathrm{T}$ scanner was used to acquire $\mathrm{T} 2 *$ weighted echo-planar images with BOLD contrast. Each echo-planar image comprised 35 axial slices of $2 \mathrm{~mm}$ thickness with $1 \mathrm{~mm}$ slice interval and $3 \times 3 \mathrm{~mm}$ inplane resolution. Volumes were acquired with an effective repetition time (TR) of $3.15 \mathrm{sec} / \mathrm{volume}$ and the first six (dummy) volumes of each run were discarded to allow for T1 equilibration effects. A total of 372 volume images were taken in two separate runs from the 11 subjects who were presented the stimuli with an SOA of $3 \mathrm{sec}$, whereas a total of 506 volume images were obtained in two separate runs from the 11 subjects who were presented the stimuli with an SOA of 4 sec. After the two functional runs, a T1-weighted anatomical volume image was acquired from all subjects.

\section{Statistical Parametric Mapping}

Statistical parametric mapping was performed using SPM2 software (Wellcome Department of Imaging Neuroscience, London, UK), running under Matlab 6.5 (Mathworks, Sherbon, MA). All volumes from each subject were realigned using the first as reference and resliced with sinc interpolation. The functional images were spatially normalized (Friston, Ashburn, et al., 1995) to a standard MNI-305 template using a total of 1323 nonlinear-basis functions. Functional data were spatially smoothed with a 6-mm full width at half maximum isotropic Gaussian kernel to compensate for residual variability in functional anatomy after spatial normalization and to permit application of Gaussian random-field theory for adjusted statistical inference.

First, the statistical analysis was performed in a subjectspecific fashion. To remove low-frequency drifts, the data were high-pass filtered using a set of discrete cosine basis functions with a cutoff period of $128 \mathrm{sec}$. Each experimental condition was modeled independently by convolving the onset times with a synthetic hemodynamic response function (HRF, with no dispersion or temporal derivatives). The parameter estimates were calculated for all brain voxels using the general linear model, and contrast images comparing each word type against false fonts (i.e., the baseline) were computed (Friston, Holmes, et al., 1995). Second, subject-specific contrast images were entered into an ANOVA to permit inferences at the population level (i.e., a random-effects analysis; Holmes \& Friston, 1998). The overall effect of reading was identified by comparing all word types against the baseline (i.e., regular, exception, and pseudo words $>$ false fonts). Word-type effects were first detected by directly comparing each word type against the others [i.e., (i) regular > exception words; (ii) exception $>$ regular words; (iii) regular $>$ pseudo words; (iv) pseudo $>$ regular words; (v) exception $>$ pseudo words; and (vi) pseudo > exception words]. Word-type effects that occurred within the reading system were then identified by performing a conjunction analysis between the overall effect of reading and each of the effects of word type independently. The $t$-images for each contrast at the second level were subsequently transformed into statistical parametric maps of the $Z$ statistic. Unless otherwise indicated, we report and discuss regions that showed significant effects at $p<.05$ (corrected for multiple comparisons across the whole brain) with an extent threshold for each cluster of 5 voxels.

\section{Dynamic Causal Modeling}

DCM uses a previously validated biophysical model of fMRI responses (Friston, Mechelli, et al., 2000) to estimate the underlying neuronal activities from the observed hemodynamic responses. The estimated underlying neuronal activities are then used to derive the connectivity parameters for the influence of experimental inputs on brain states, for the intrinsic connections, and for the bilinear terms (i.e., changes in connectivity induced by experimental factors). These two steps are repeated iteratively and correspond to the expectation and maximization steps of an EM algorithm. The intrinsic connections estimate the rate of change of neuronal activity in one area that is associated with activity in another. As such, this characterization does not depend on the units of activity per se, but the "speed" or rate of interregional coupling.

For each subject, a dynamic causal model was constructed which included seven left-lateralized regions of interest. These included the pars triangularis [mean coordinates $(x, y, z):-52,34,4]$, the pars opercularis [mean coordinates $(x, y, z):-56,10,4]$, and the dorsal premotor cortex [mean coordinates $(x, y, z):-52,-8$, 44], all of which expressed word-type-sensitive activations in the present study. Posterior regions of interest included the posterior fusiform [mean coordinates $(x$, $y, z):-48,-64,-18$ ], middle fusiform [mean coordinates $(x, y, z):-46,-54,-18]$, anterior fusiform [mean coordinates $(x, y, z):-44,-44,-16]$, and superior temporal cortex [mean coordinates $(x, y, z)$ : $-56,-44,4]$. These regions were defined as $6-\mathrm{mm}$ radius spheres centered on maxima of the subjectspecific statistical parametric maps for the overall effect of reading relative to false fonts (i.e., regular, exception, and pseudo words $>$ false fonts). Regional activities were defined as the principal eigenvariate from each region of interest, in a subject-specific fashion. A stimulus function that encoded the visual presentation 
of all words entered the dynamic causal model through the three fusiform areas in agreement with a current anatomical model of reading (Price, Gorno-Tempini, et al., 2003). The resulting perturbation was then allowed to propagate throughout the network via interconnections between the three fusiform areas and the remaining regions. The dynamic causal model comprised forward and backward "intrinsic connections" between the three fusiform areas and the superior temporal area; between the superior temporal area and the three prefrontal areas; and between the three fusiform areas and the three prefrontal areas. "Bilinear terms" were also specified to examine the influence of word type on all backward and forward connections in the network. This influence was encoded by boxcar functions, indicating the word type (regular, exception, and pseudo words).

The forward and backward "intrinsic connections" (which characterize the coupling between regions irrespective of word type) and the "bilinear terms" (which capture how the intrinsic connections vary as a function of word type) were estimated for each subject independently. The sort of fit that we observed typically is represented in Figure 4.
The estimated values for the intrinsic connections refer to the effective connectivity (i.e., the effect of one region over another), as opposed to the underlying anatomical connectivity. Likewise, the estimated values for the bilinear terms refer to changes in effective connectivity rather than anatomical connectivity. The ensuing subject-specific parameters were taken to a second level for population inference (Holmes \& Friston, 1998). Thus, the present DCM investigation identified those neuronal interactions that were relatively consistent across subjects. This does not exclude the possibility that some connections also expressed significant variability across subjects, as previously demonstrated using a similar paradigm (Mechelli, Penny, Price, \& Friston, 2002). Statistical tests were performed to examine the coupling between regions irrespective of word type as well as changes in strength for regular, exception, and pseudo words, respectively. These were simple one-sample $t$ tests on the intrinsic connections and bilinear terms. As this was repeated for several connections, we adopted a conservative statistical threshold of $p<.001$ (equivalent to a Bonferroni correction for 50 tests with a statistical threshold of $p<.05)$.

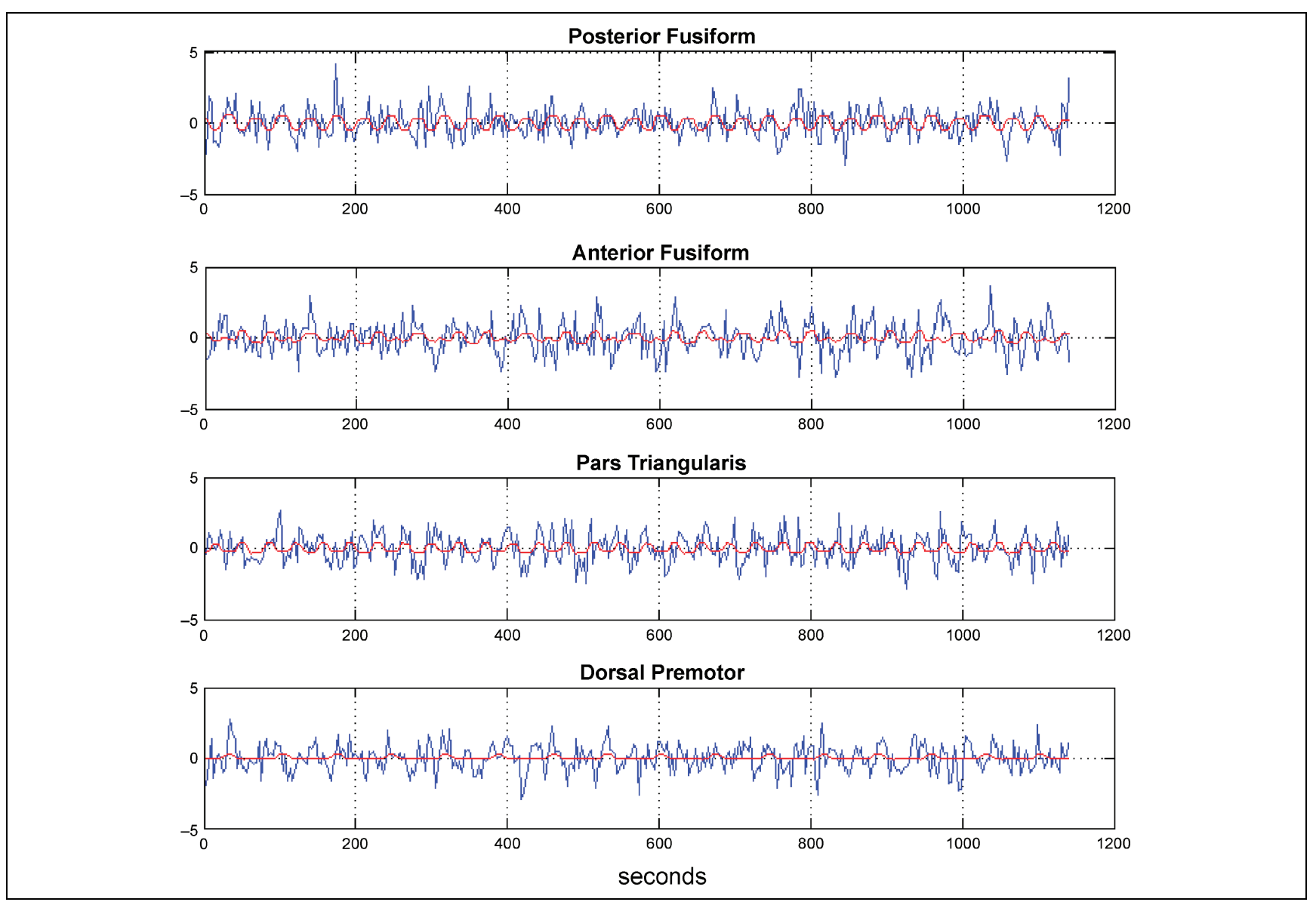

Figure 4. Fitted responses in four regions of interest in a typical subject. The blue line represents the observed BOLD response (as indexed by the principal eigenvariate); the red line represents the BOLD response predicted by the DCM model. 


\section{Acknowledgments}

AM is supported by MH64445 from the National Institutes of Health (USA). JC, KJF, and CJP are supported by The Wellcome Trust.

Reprint requests should be sent to Andrea Mechelli, Wellcome Department of Imaging Neuroscience, 12 Queen Square London, WC1N 3BG, UK, or via e-mail: andream@fil.ion.ucl.ac.uk.

The data reported in this experiment have been deposited with the fMRI Data Center archive (www.fmridc.org). The accession number is 2-2005-118M7.

\section{REFERENCES}

Binder, J. R., McKiernan, K. A., Parsons, M. E., Westbury, C. F., Possing, E. T., Kaufman, J. N., \& Buchanan, L. (2003). Neural correlates of lexical access during visual word recognition. Journal of Cognitive Neuroscience, 15, 372-393.

Bokde, A. L., Tagamets, M. A., Friedman, R. B., \& Horwitz, B. (2001). Functional interactions of the inferior frontal cortex during the processing of words and word-like stimuli. Neuron, 30, 609-617.

Brunswick, N., McCrory, E., Price, C. J., Frith, C. D., \& Frith, U. (1999). Explicit and implicit processing of words and pseudowords by adult developmental dyslexics: A search for Wernicke's Wortschatz? Brain, 122, 1901-1917.

Coltheart, M. (1981). MRC psycholinguistic database user manual: Version 1. Birkbeck College, London, UK.

Coltheart, M., Rastle, K., Perry, C., Laugdon, R., \& Ziegler, J. (2001). DRC: A dual route cascaded model of visual word recognition and reading aloud. Psychological Review, 108, 204-256.

Crisp, J., \& Lambon Ralph, M. A. (in press). Unlocking the nature of the phonological-deep dyslexia continuum: The keys to reading aloud are in phonology and semantics. Journal of Cognitive Neuroscience.

Damasio, A. R. (1989). Time-locked multiregional retroactivation: A systems-level proposal for the neural substrates of recall and recognition. Cognition, 33, 25-62.

Devlin, J. T., Matthews, P. M., \& Rushworth, M. F. S. (2003). Semantic processing in the left inferior prefrontal cortex: A combined functional magnetic resonance imaging and transcranial magnetic stimulation study. Journal of Cognitive Neuroscience, 15, 71-84.

Fiebach, C. J., Friederici, A. D., Müller, K., \& von Cramon, D. Y. (2002). fMRI evidence for dual routes to the mental lexicon in visual word recognition. Journal of Cognitive Neuroscience, 14, 11-23.

Fiez, J., Katz, W., \& Tallal, P. (1995). Studies of auditory and phonological processing: Effects of stimulus characteristics and task demands. Journal of Cognitive Neuroscience, 7 , 357-375.

Fiez, J. A., Balota, D. A., Raichle, M. E., \& Petersen, S. E. (1999). Effects of lexicality, frequency, and spelling-to-sound consistency on the functional anatomy of reading. Neuron, 24, 205-218.

Friston, K. J., Ashburner, J., Frith, C. D., Poline, J.-B., Heather, J. D., \& Frackowiak, R. S. J. (1995). Spatial registration and normalization of images. Human Brain Mapping, 2, $1-25$.

Friston, K. J., Harrison, L., \& Penny, W. (2003). Dynamic causal modelling. Neuroimage, 19, 1273-1302.

Friston, K. J., Holmes, A., Worsley, K. J., Poline, J.-B., Frith, C. D., \& Frackowiak, R. S. J. (1995). Statistical parametric maps in functional imaging: A general linear approach. Human Brain Mapping, 2, 189-210.

Friston, K. J., Mechelli, A., Turner, R., \& Price, C. J. (2000). Nonlinear responses in fMRI: The balloon model, Volterra kernels and other hemodynamics. Neuroimage, 12, 466-477.

Friston, K. J., \& Price, C. J. (2001). Dynamic representations and generative models of brain function. Brain Research Bulletin, 54, 275-285.

Graham, N. L., Patterson, K., \& Hodges, J. R. (2000). The impact of semantic memory impairment on spelling: Evidence from semantic dementia. Neuropsychologia, 38, 143-163.

Hagoort, P., Brown, C., Indefrey, P., Herzog, H., Steinmetz, H., \& Seitz, R. (1999). The neural circuitry involved in the reading of German words and pseudowords: A PET study. Journal of Cognitive Neuroscience, 11, 383-398.

Herbster, A. N., Mintun, M. A., Nebes, R. D., \& Becker, J. T. (1997). Regional cerebral blood flow during word and nonword reading. Human Brain Mapping, 5, 84-92.

Hodges, J. R., Patterson, K., Oxbury, S., \& Funnell, E. (1992). Semantic dementia: Progressive fluent aphasia with temporal lobe atrophy. Brain, 115, 1783-1806.

Holmes, A. P., \& Friston, K. J. (1998). Generalisability, random effects and population inference. Neuroimage, 7, S754.

Kucera, H., \& Francis, W. H. (1967). Computational analysis of present-day American English. Providence, RI: Brown University Press.

Lambon Ralph, M. A., \& Graham, N. L. (2000). Previous cases: Acquired phonological and deep dyslexia. Neurocase, 6, 141-178.

McCandliss, B. D., Cohen, L., \& Dehaene, S. (2003). The visual word form area: Expertise for reading in the fusiform gyrus. Trends in Cognitive Sciences, 7, 293-299.

McDermott, K. B., Petersen, S. E., Watson, J. M., \& Ojemann, J. G. (2003). A procedure for identifying regions preferentially activated by attention to semantic and phonological relations using functional magnetic resonance imaging. Neuropsychologia, 41, 293-303.

McIntosh, A. R. (2000). Towards a network theory of cognition. Neural Networks, 13, 861-870.

Mechelli, A., Friston, K. J., \& Price, C. J. (2000). The effects of presentation rate during word and pseudoword reading: A comparison of PET and fMRI. Journal of Cognitive Neuroscience, 12(Suppl. 2), 145-156.

Mechelli, A., Gorno-Tempini, M. L., \& Price, C. J. (2003). Neuroimaging studies of word and pseudoword reading: Consistencies, inconsistencies, and limitations. Journal of Cognitive Neuroscience, 15, 260-271.

Mechelli, A., Penny, W. D., Price, C. J., \& Friston, K. J. (2002). Effective connectivity and intersubject variability: Using a multisubject network to test differences and commonalities. Neuroimage, 17, 1459-1469.

Mechelli, A., Price, C. J., \& Friston, K. J. (2001). Nonlinear coupling between evoked rCBF and BOLD signals: A simulation study of hemodynamic responses. Neuroimage, 14, 862-872.

Mechelli, A., Price, C. J., Friston, K. J., \& Ishai, A. (2004). Where bottom-up meets top-down: Neuronal interactions during perception and imagery. Cerebral Cortex, 14, 1256-1265.

Mechelli, A., Price, C. J., Noppeney, U., \& Friston, K. J. (2003). A dynamic causal modelling study of category effects: Bottom-up or top-down mediation? Journal of Cognitive Neuroscience, 15, 925-934.

Mesulam, M. M. (1990). Large-scale neurocognitive networks and distributed processing for attention, language, and memory. Annals of Neurology, 28, 597-613. 
Mummery, C. J., Patterson, K., Hodges, J. R., \& Price, C. J. (1998). Functional neuroanatomy of the semantic system: Divisible by what? Journal of Cognitive Neuroscience, 10, 766-777.

Noppeney, U., \& Price, C. J. (2002). Retrieval of visual, auditory, and abstract semantics. Neuroimage, 15, 917-926.

Patterson, K., \& Hodges, J. R. (1992). Deterioration of word meaning: Implications for reading. Neuropsychologia, 30, 1025-1040.

Patterson, K., \& Lambon Ralph, M. A. (1999). Selective disorders of reading? Current Opinion in Neurobiology, 9, 235-239.

Patterson, K., \& Marcel, A. J. (1992). Phonological alexia of phonological alexia? In J. Alegria, D. Holender, J. Junca de Morais, \& M. Radeau (Eds.), Analytic approaches to buman cognition (pp. 259-274). Amsterdam: Elsevier Science Publishers.

Patterson, K., Suzuki, T., \& Wydell, T. (1996). Interpreting a case of Japanese phonological alexia: The key is phonology. Cognitive Neuropsychology, 13, 803-822.

Paulesu, E., McCrory, E., Fazio, F., Menoncello, L., Brunswick, N., Cappa, S. F., Cotelli, M., Cossu, G., Corte, F., Lorusso, M., Pesenti, S., Gallagher, A., Perani, D., Price, C., Frith, C. D., \& Frith, U. (2000). A cultural effect on brain function. Nature Neuroscience, 3, 91-96.

Plaut, D. C., McClelland, J. L., Seidenberg, M., \& Patterson, K. (1996). Understanding normal and impaired word reading: Computational principles in quasi-regular domains. Psychological Review, 103, 56-115.

Price, C. J., \& Devlin, J. T. (2003). The myth of the visual word form area. Neuroimage, 19, 473-481.

Price, C. J., \& Friston, K. J. (1999). Scanning patients with tasks they can perform. Human Brain Mapping, 8, 102-108.
Price, C. J., Gorno-Tempini, M. L., Graham, K. S., Biggio, N., Mechelli, A., Patterson, K., \& Noppeney, U. (2003). Normal and pathological reading: Converging data from lesion and imaging studies. Neuroimage, 20 (Suppl. 1), 30-41.

Price, C. J., Wise, R. J. S., \& Frackowiak, R. S. J. (1996). Demonstrating the implicit processing of visually presented words and pseudowords. Cerebral Cortex, 6, 62-70.

Roskies, A. L., Fiez, J. A., Balota, D. A., Raichle, M. E., \& Petersen, S. E. (2001). Task-dependent modulation of regions in the left inferior frontal cortex during semantic processing. Journal of Cognitive Neuroscience, 13, 829-843.

Rumsey, J. M., Horwitz, B., Donohue, C., Nace, K., Maisog, J. M., \& Andreason, P. (1997). Phonological and orthographic components of word recognition: A PET-rCBF study. Brain, 120, 739-759.

Tagamets, M.-A., Novick, J. M., Chalmers, M. L., \& Friedman, R. B. (2000). A parametric approach to orthographic processing in the brain: An fMRI study. Journal of Cognitive Neuroscience, 12, 281-297.

Turkeltaub, P. E., Eden, G. F., Jones, K. M., \& Zeffiro, T. A. (2002). Meta-analysis of the functional neuroanatomy of single word reading: Method and validation. Neuroimage, 16, 765-780.

Xu, B., Grafman, J., Gaillard, W. D., Ishii, K., Vega-Bermudez, F., Pietrini, P., Reeves-Tyler, P., DiCamillo, P., \& Theodore, W. (2001). Conjoint and extended neural networks for the computation of speech codes: The neural basis of selective impairment in reading words and pseudowords. Cerebral Cortex, 11, 267-277.

Zatorre, R. J., Evans, A. C., Meyer, E., \& Gjedde, A. (1992). Lateralization of phonetic and pitch discrimination in speech processing. Science, 256, 846-849. 